An Analytical Solution of Macrodispersivity for Adsorbing Solute Transport in Unsaturated Soils

Jinzhong Yang, Renduo Zhang and Jinquan Wu

Journal Article $1996 \quad$ WWRC-96-02

Water Resources Research, 32(2): 355-362

Jinzhong Yang, Renduo Zhang and Jinquan Wu

Department of Plant, Soil, and Insect Sciences

University of Wyoming

Laramie, Wyoming 


\title{
An analytical solution of macrodispersivity for adsorbing solute transport in unsaturated soils
}

\author{
Jinzhong Yang, ${ }^{1}$ Renduo Zhang, and Jinquan Wu \\ Department of Plant. Suil, and Insect Sciences, University of Wyoming. Laramie
}

\begin{abstract}
An analytical solution of macrodispersivity was derived for the adsorbing solute transport in physically and chemically heterogeneous unsaturated soils under the condition of gravity-dominated flow. The unsaturated hydraulic conductivity and water content were treated as spatial random functions. The chemical adsorption was described by a linear equilibrium isotherm, and the adsorption coefficient was represented as a spatial random function with specified correlation structures. The closed-form expression for macrodispersivity was expressed as a function of the hydraulic and adsorbing statistical properties of the unsaturated soil. For a short solute travel distance the macrodispersivity linearly increased with the increase of the solute plume travel distance. The macrodispersivity approached an asymptotic value when the center of the solute plume moved a long distance. The general solution quantified the effects of various factors, such as variabilities of the water content and unsaturated hydraulic conductivity, mean water content. and the retardation factor. on the macrodispersivity.
\end{abstract}

\section{Introduction}

Experimental evidence and theoretical results suggest that solute spreading in the natural field is governed by the spatial variability of soil properties. Due to the spatial variability. the dispersivity measured at the laboratory scule cannot be used for the prediction of solute distributions at the field scale. which is of most interest for environmental upplicutions. Stochastic models have been developed to represent the transport phenomenon of nonreactive solute at the large scale [e.g.. Dagan. 19K2: Gelhar and Axness, 1983]. Those theoretical studies reveal that the average solute concentration can be described by the convection-dispersion equation with an apparent dispersion coefficient that relates to the spatial variability of the saturated hydraulic conductivity field. Under steady state water flow and an uniform hydraulic gradient. Dagan [1984] obtains a closed-form expression of the dispersion coefficient in saturated soils. The longitudinal dispersion coefficient tends to a constant and the transverse dispersion coefficient tends to zero when the solute plume travels tens of the correlation scale of log-transformed saturated hydraulic conductivity. Field experiments for solute transport in the saturated soil showed a good agreement between solute spreading and spatial moments with theoretical analyses [Freyberg, 1986].

Many contaminants commonly encountered in the groundwater system undergo interactions with porous media. The solute transport processes become much more complicated when the solute moves through a heterogeneous medium with spatial variability of chemical properties [Roberts et al., 1986]. Analytical solutions of reactive solute transport in saturated media demonstrate that the field dispersion coefficient relates

\footnotetext{
'Also at Department of Hydraulic Engineering, Wuhan University of Hydraulic and Electric Engineering, Wuhan, Hubei, People's Republic of China.

Copyright 1996 by the American Geophysical Union.

Paper number 95WR03488.

0043-1397/96/95WR-03488\$05.00
}

to the retardation factor and first-order decay coefficient. and the retardation factor has a large impact on the longitudinal dispersion [Kabala and Sposito. 1991: Bellin et al.. 1993].

Groundwater contamination mav result from surface contaminant spills or agricultural practices. Before contaminants reach the groundwater table. they move through and react with the unsaturated soil. Therefore yuantitative description of chemical transport in the unsaturated zone is important for the management of potential hazardous chemicals in the ecosystem. Water flow is much more intricate in the unsaturated zone than in the saturated zone. Besides the high heterogeneity of the unsaturated soil. soil hydraulic functions. such as the unsaturated conductivity and the soil water capacity, depend on both soil properties and the soil water content or pressure head. Russo [1993] analyzes solute dispersion in heterogeneous unsaturated soils by using a general Lagrangian formulation related the stochastic theory of Yeh et al. [1985] for steady water flow. Because of the complexity of solute transport under unsaturated flow conditions, it is difficult to obtain a closedform expression of the macrodispersivity. To our knowledge. little work has been done on solute transport adsorption in physically and chemically heterogeneous unsaturated soils.

In this paper we address the problem of combined effects of adsorbing solute transport in unsaturated heterogeneous soil. Under the gravity-dominated unsaturated flow, a closed-form solution of macrodispersivity was derived and expressed as a function of statistical properties of the unsaturated soil and chemical heterogeneities. The effects of various factors on the macrodispersivity were discussed based on the solution.

\section{Theory}

By using the general Lagrangian formulation and neglecting the pore scale dispersion, statistical properties of the solute particle velocity are related to the first- and second-order moments by [Dagan, 1984]

$$
d\langle X\rangle / d t=\langle U\rangle
$$




$$
\frac{1}{2} \frac{d M_{n}}{d t}=\int_{0}^{t} C_{i j}\left(\langle U\rangle t^{\prime}\right\rangle d t^{\prime}
$$

Here $\langle X\rangle$ is the expected value of the particle position, $M_{i j}$ is the component of the second-order spatial moments of the solute body, $\langle U\rangle$ is the average velocity of a solute particle. $C_{i j}(\mathbf{r})=\left\langle U_{i}^{\prime}(\mathbf{x}) U_{j}^{\prime}(\mathbf{x}-\mathbf{r})\right\rangle$ is the component of the cross covariance of solute velocities $U_{i}$ and $U_{j} . U_{i}^{\prime}(\mathbf{x})=U_{i}(\mathbf{x})-$ $\left\langle U_{i}(\mathbf{x})\right\rangle$ is the perturbation of $U_{i}(\mathbf{x})$ from its mean $\left\langle U_{i}(\mathbf{x})\right\rangle$, and $\mathbf{r}$ is the separation vector. The macrodispersion coefficient $D_{i j}$ can be related to the second-order moment by [Aris, 1956]

$$
D_{i !}=\frac{1}{2} \frac{d M_{i t}}{d t}
$$

For the unsaturated soil. the soil water flux is expressed as

$$
q_{i}=K(\psi) \frac{\partial\left(\psi+x_{1}\right)}{\partial x_{i}}
$$

where $K(\psi)$ is the unsaturated hydraulic conductivity, $\psi$ is the suction head, and $x_{1}$ is the vertical coordinate, positive downward. When the chemical reaction with soil is a linear equilibrium type. the adsorbed concentration $C$, is related to the soil water concentration $C$ by

$$
C_{,}(\mathbf{x} . t)=\frac{\rho K_{t}(\mathbf{x})}{\theta} C(\mathbf{x} . t)
$$

where $\rho$ is the soil bulk density, $K_{d}(\mathbf{x})$ is the adsorption or distribution coefficient. and $\theta$ is the volumetric water content. By defining the retardation factor as

$$
R=1-\rho K_{d}^{\prime}(\mathbf{x}) ; \theta
$$

the solute velocity $C^{*}=\left(L_{1}, L_{2} . U_{3}\right)$ is given by

$$
L_{i}=q_{i} y \quad y=\theta R \quad i=1,2.3
$$

The transport process of a linear equilibrium adsorption solute can be described by a convection-dispersion equation with the velocity $U$.

By expressing $\psi$ in terms of its mean and perturbation. i.e..

$$
\psi=H+h \quad\langle h\rangle=0
$$

the soil water flux can be written as

$$
q_{1}=K(\psi)\left[\frac{\partial\left(H-x_{1}\right)}{\partial x_{i}}+\frac{\partial h}{\partial x_{i}}\right]=K(\psi)\left[J_{i}+\frac{\partial h}{i x_{i}}\right]
$$

where $J_{i}=\partial\left(H+x_{1}\right) i \dot{i} x_{i}$ is the mean hydraulic gradient in the direction $x_{i}$. For the sake of simplicity it is assumed that the mean hydraulic gradient exists only in the vertical direction. i.e.. $J_{i}=(J, 0,0)$.

Under the condition of gravity-dominated flow, the mean hydraulic gradient in (9) tends to 1 , and the variance tends to 0 . This condition is supported by numerical and analytical results. In a numerical analysis of unsaturated gravitydominated flow, Russo [1991] finds that the mean hydraulic gradient $J$ is 1 and the variance $\sigma_{J}^{2}$ is 0.001 . From a stochastic analysis of water flow in unsaturated soils. Ych et al. [1985] obtain $\sigma_{J}^{2}=0.04-0.2 \sigma_{f}^{2} J$ for most soils. Here $\sigma_{f}^{2}$ is the variance of log-transformed saturated hydraulic conductivity. Taking $\sigma_{f}^{2}=0.3$ and $J=1$. we have $\sigma_{j}^{2}=0.001-0.06$. Therefore (9) can be simplified to

$$
q_{i}=K(\psi) J_{i}=(q, 0,0)
$$

where $q=K(\psi)$ is the soil water flux in the vertical direction. $K(\psi), \theta(\psi), K_{d}(\mathbf{x})$, and $R$ are expressed in terms of their mean values and perturbations, i.e.,

$$
\begin{aligned}
& K(\psi)=\langle K\rangle+K^{\prime}\left\langle K^{\prime}\right\rangle=0 \\
& \theta(\psi)=\langle\theta\rangle+\theta^{\prime}\left\langle\theta^{\prime}\right\rangle=0 \\
& K_{d}(X)=\left\langle K_{d}\right\rangle+K_{d}^{\prime} \quad\left\langle K_{d}^{\prime}\right\rangle=0 \\
& y=\langle y\rangle+y^{\prime} \quad\langle y\rangle=\langle\theta\rangle+\rho\left\langle K_{d}\right\rangle \quad y^{\prime}=\theta^{\prime}+\rho K_{d}^{\prime}
\end{aligned}
$$

Similarly, the soil water flux can be written as

$$
q=\langle q\rangle+q^{\prime} \quad\langle q\rangle=\langle K\rangle \quad q^{\prime}=K^{\prime}
$$

From (7), (11), and (12) the solute velocity can be described by

$$
U=\frac{\langle q\rangle+q^{\prime}}{\langle y\rangle+y^{\prime}}=\left(\langle q\rangle+q^{\prime}\right)\left(\frac{1}{\langle y\rangle}-\frac{y^{\prime}}{\langle y\rangle^{2}}+\frac{y^{\prime 2}}{\langle y\rangle^{3}}-\frac{y^{\prime 3}}{\langle y\rangle^{4}}+\cdots\right)
$$

Neglecting the perturbation terms higher than first order. we have

$$
\langle U\rangle=\langle q\rangle /\langle v\rangle
$$

Subtracting (14) from (13) and neglecting perturbation terms higher than first order. we have the perturbation of the solute velocity

$$
L^{\prime \prime}=\frac{(q)}{(y)}\left(\frac{q^{\prime}}{\langle q\rangle}-\frac{y^{\prime}}{\langle(y)}\right)
$$

The covariance function of the solute velocity is determined from (15) as

$$
\begin{aligned}
C_{L U^{\prime}}(\mathbf{r}) & =\left\langle U^{\prime}(\mathbf{x}) U^{\prime}(\mathbf{x}-\mathbf{r})\right\rangle \\
& =\frac{\langle q\rangle^{2}}{\langle v\rangle^{2}}\left[\frac{C_{q q}(\mathbf{r})}{\langle q\rangle^{2}}-\frac{C_{w l}(\mathbf{r})+C_{v q}(-\mathbf{r})}{\langle q\rangle\langle v\rangle}-\frac{C_{n s}(\mathbf{r})}{\langle v\rangle^{2}}\right]
\end{aligned}
$$

By using (11) and (12), the covariance and cross covariance of $q$ and $y$ in (16) are calculated by

$$
\begin{gathered}
C_{q q}(\mathbf{r})=C_{K K}(\mathbf{r}) \\
C_{y q}(\mathbf{r})=C_{\theta K}(\mathbf{r})+\rho C_{K_{i k}}(\mathbf{r}) \\
C_{y,}(\mathbf{r})=C_{\theta A}(\mathbf{r})+\rho\left[C_{\theta K_{i}}(\mathbf{r})+C_{\theta K_{i}}(-\mathbf{r})\right]+\rho^{2} C_{K, K}(\mathbf{r})
\end{gathered}
$$

It is assumed that the unsaturated hydraulic conductivity $K(\mathbf{x} . H)$ can be described by [Gardner, 1958]

$$
K(\mathbf{x}, H)=K_{s}(\mathbf{x}) \exp [-\alpha(\mathbf{x}) H]
$$

where $K_{s}(\mathbf{x})$ is the saturated hydraulic conductivity. $\alpha$ is the soil parameter related to the soil pore size distribution. and $H$ is the mean suction head. The analyses of Freeze [1975] and Sudichy [1986] suggest that the distribution of the saturated conductivity be lognormal for most soils. It is assumed that the parameter $\alpha$ is a normal distribution. Both $\ln K_{s}$ and $\alpha$ are regarded as realizations of stationary second-order spatial random functions (SRFs) with anisotropic covariance functions of the form

$C_{\eta \eta}(\mathbf{r})=\sigma_{\eta}^{2} \exp \left\{-\left[\left(\frac{r_{1}}{\lambda_{\eta 1}}\right)^{2}+\left(\frac{r_{2}}{\lambda_{\eta 2}}\right)^{2}+\left(\frac{r_{3}}{\lambda_{\eta ! 3}}\right)^{2}\right]^{1 / 2}\right\}$ 
where $\eta$ indicates $\ln \left(K_{s}\right)$ or $\alpha: \sigma_{\eta}^{2}$ is the variance of $\eta ; r_{1}, r_{2}$, and $r_{3}$ are the components of the separation vector $\mathbf{r}$; and $\lambda_{\eta 1}$, $\lambda_{\eta 2}$, and $\lambda_{\eta 3}$ are the correlation lengths of $\eta$ in the $x_{1}, x_{2}$, and $x_{3}$ directions: respectively. Russo and Bouton [1992] indicate that the correlation between $\ln K_{s}$ and $\alpha$ is weak and they can be considered as the uncorrelated SRFs. Corresponding to the model of the unsaturated hydraulic conductivity (equation (20)), the water content is expressed as [Russo, 1988]

$$
\begin{gathered}
\theta(\mathbf{x} . H)=\theta_{r}+\left\{\theta_{1}-\theta_{r}\right)\left\{\exp \left[-\frac{1}{2} \alpha(\mathbf{x}) H\right]\right. \\
\left.\cdot\left[1+\frac{1}{2} \alpha(\mathbf{x}) H\right]\right\}^{2 \cdot m+2}
\end{gathered}
$$

where $\theta_{s}$ and $\theta_{r}$ are the saturated and residual water contents. respectively, $m$ is a parameter. Expressing $\ln K_{\text {s }}$ and $\alpha$ by the mean values and thuctuations, i.e..

$$
\begin{aligned}
\ln \left(K_{s}\right) & =F+f & \langle f\rangle & =0 \\
\alpha & =A+a & \langle a\rangle & =0
\end{aligned}
$$

we have

$$
\begin{gathered}
\ln [K(\mathbf{x}, H)]=\ln \left[K_{1}\right]-\alpha H=p=\langle p\rangle+p^{\prime} \\
\langle p\rangle=F-A H \quad p^{\prime}=f-a H \quad\left\langle p^{\prime}\right\rangle=0
\end{gathered}
$$

The unsaturated hydraulic conductivity is expressed as

$$
\begin{aligned}
K(\mathbf{x} . H)=\exp [p(\mathbf{x}, H)] & =K^{\prime \prime} \exp \left[p^{\prime}(\mathbf{x} . H)\right] \\
K^{\prime \prime} & =e^{\prime \prime}=K_{e^{\prime}}^{\prime \prime H} \quad K^{\prime \prime}=e^{\prime}
\end{aligned}
$$

Here. $p=\ln (K)$ is also a normal SRF because it is the sum of two normal SRFs.

Studies of solute transport in the laboratory and tield $\{$ Roherts et al.. 1986) indicate that the reaction of solute with soil. such as adsorption. is a spatial variable. Therefore the spatial distribution of the adsorption coefficient is considered to be a SRF. Plausible variability of adsorption is assumed to be either correlated or uncorrelated with the log-transformed saturated hydraulic conductivity [Destouni and Cretkovic, 1991: Bellin et al. 1993: Bosma et al., 1993]. If there is no correlation between the adsorption coefficient and the saturated hydraulic conductivity, the heterogeneity of the adsorption coefficient can be described as

$$
K_{d}(\mathbf{x})=K_{d}^{G} \exp \left[N_{d}(\mathbf{x})\right]
$$

where $K_{d}^{G}$ is the geometric mean of $K_{d}(\mathbf{x})$ and $N_{d}(\mathbf{x})$ is a normal SRF with a mean 0 and variance $\sigma_{d}^{2}$. The correlation function of $N_{d}(\mathbf{x})$ is described by an exponential form similar to (21). A perfect correlation between $\ln K_{d}$ and $\ln K_{s}$ is given by

$$
K_{d}(\mathbf{x})=K_{d}^{G} \exp [\beta f(\mathbf{x})]
$$

For the positive (or negative) correlation between $\ln K_{c}$ and $\ln$ $K_{\text {s }}, \beta$ is greater (or less) than 0 .

Substituting $\alpha=A+a$ into (22) and retaining the firstorder term of $a$, the water content can be expressed as a lognormal SRF

$$
\theta=\theta_{r}+\left(\theta_{s}-\theta_{r}\right) e^{E+B a}
$$

where

$$
\begin{gathered}
E=\frac{2}{m+2}\left[-\frac{1}{2} A H+\ln \left(1+\frac{1}{2} A H\right)-\frac{1}{2}\left(\frac{H \sigma_{a}}{2+A H}\right)^{2}\right] \\
B=\frac{-A H^{2}}{(m+2)(A H+2)}
\end{gathered}
$$

If $X(\mathrm{~s})$ and $Y(\mathrm{~s})$ are nurmal stationary SRFs, the crosscovariance function of $\xi=\exp [X(\mathrm{~s})]$ and $\eta=\exp [b Y(\mathrm{~s})]$ is [Christakios. 1992]

$$
\begin{aligned}
C_{i \eta}(\mathbf{r})= & \exp \left[\langle X\rangle+b\langle Y\rangle+\frac{1}{2}\left(\sigma_{Y}^{2}+b^{2} \cdot \sigma_{Y}^{2}\right)\right] \\
& \cdot\left\{\exp \left[b C_{n Y}(\mathbf{r})\right]-1\right\}
\end{aligned}
$$

where $b$ is a constant, $\langle X\rangle$ and $\langle Y\rangle$ are the mean values, $\sigma_{X}^{2}$ and $\sigma_{Y}^{2}$ are the variances. and $C_{Y Y}$ is the cross covariance of $X(\mathrm{~s})$ and $Y(s)$. From (24) and in the case of noncorrelation between In $K$, and $\alpha$, the covariance of $p=\ln K(\mathbf{x} . H)$ can be written in the form

$$
C_{p p}(\mathbf{r})=C_{i f}(\mathbf{r})+H^{2} C_{u u}(\mathbf{r})
$$

By using (30), the covariance of water Hux $q$ is determined from (17), (24), (25), and (31) as

$$
\begin{aligned}
C_{u q}(\boldsymbol{r})= & \left(K^{(i)}\right)^{2} \exp \left[\sigma_{f}^{2}+H^{i} \sigma_{u}^{i}\right] \\
& \cdot\left\{\exp \left[C_{u t}(\mathbf{r})-H^{i} C_{u}(\mathbf{r})\right]-1\right\}
\end{aligned}
$$

The covariance of water content and the cross covariance of water content with unsaturated hydraulic conductivity are ohtained from (24). (28), and (30); i.e.,

$$
\begin{aligned}
& C_{H H}(\mathbf{r})=\left(H_{0}-H_{r}\right)^{2} \exp \left(2 E+B^{2} \sigma_{u}^{2}\right) \\
& \cdot\left\{\exp \left[B^{*} C_{. u t}(\mathbf{r})\right]-1\right\} \\
& C_{H K}(\mathbf{r})=\left(H-H_{H}\right)(K) \exp \left(E+\frac{1}{2} B^{2} \sigma_{u}^{2}\right) \\
& \cdot\left\{\exp \left[-B H C_{c u}(\mathbf{r})\right]-1\right\}
\end{aligned}
$$

For the perfect correlation between $\ln K_{d}$. and $\ln K_{s}$, from (24). (27), and (30) we have

$$
\begin{array}{r}
C_{K_{i} K}(\mathbf{r})=K_{i l}^{(i} K^{i j} \exp \left[\frac{1}{2}\left(\beta^{2} \sigma_{f}^{2}+\sigma_{f}^{2}+H^{2} \sigma_{a}^{2}\right)\right] \\
\cdot\left\{\exp \left[\beta C_{i f}(\mathbf{r})\right]-1\right\} \\
C_{K i K_{i}}(\mathbf{r})=\left(K_{d j}^{(j)}\right)^{2} \exp \left[\beta^{2} \sigma_{f}^{2}\right]\left\{\exp \left[\beta^{2} C_{f f}(\mathbf{r})\right]-1\right\}
\end{array}
$$

If $\ln K_{d}$ is not correlated with $\ln K_{s}$, the covariance and crosscovariance functions are calculated from (24), (26), and (30) as

$$
\begin{gathered}
C_{K_{d} K}(\mathbf{r})=0 \\
C_{K_{i} K_{d}}(\mathbf{r})=\left(K_{d}^{(i)}\right)^{2} \exp \left[\sigma_{d}^{2}\right]\left\{\exp \left[C_{d d}(\mathbf{r})\right]-1\right\}
\end{gathered}
$$

where $\sigma_{d}^{2}$ and $C_{d d}$ are the variance and covariance of the perturbation of $\ln K_{\iota}$. The longitudinal macrodispersivity may be defined as $A_{z:=}=D_{z:=} /\langle U\rangle$. Then from (2) and (3) we obtain

$$
A_{::}=\frac{1}{\langle U\rangle} \int_{1)}^{t} C_{U U^{\prime}}\left(\langle U\rangle t^{\prime}\right) d t^{\prime}=\frac{1}{\langle U\rangle^{2}} \int_{0}^{z_{t}} C_{U U}(\xi) d \xi
$$

where $z_{c}=\langle U\rangle_{t}$ is the mean travel distance of the solute plume. By substituting (16) into (39) and assuming no correlation between $\theta$ and $K_{d}$, the macrodispersivity $A: z$ is then described by 


$$
A_{: 2}=A_{: 1}+A_{: 2}+A_{z 3}
$$

where

$$
\begin{aligned}
& A_{z 1}= \int_{0}^{=}\left\{\exp \left[C_{f f}(\xi)+H^{2} C_{a a}(\xi)\right]-1\right\} d \xi \\
&-\int_{i:}^{:} \frac{1}{\langle q\rangle\langle y\rangle}\left[C_{\theta K}(\xi)+C_{\theta K}(-\xi)\right] d \xi \\
&+\int_{: 1}^{=} \frac{C_{\theta \theta}(\xi)}{\langle y\rangle^{2}} d \xi \\
&\langle q\rangle=\langle K\rangle=K^{G} \exp \left[\frac{1}{2}\left(\sigma_{f}^{2}+H^{2} \sigma_{a}^{2}\right)\right]
\end{aligned}
$$

For perfect correlation between $\ln K_{d}$ and $\ln K_{s}, A_{z 2}$ and $A_{z 3}$ have the forms of

$$
\begin{aligned}
& A_{z: 2}=-\int_{11}^{:} 2\left(1-\frac{1}{\langle R\rangle}\right)\left\{\exp \left[\beta C_{f f}(\xi)\right]-1\right\} d \xi \\
& A_{z:=}=\int_{11}^{:}\left(1-\frac{1}{\langle R\rangle}\right)^{2}\left\{\exp \left[\beta^{2} C_{, f}(\xi)\right]-1\right\} d \xi
\end{aligned}
$$

where $\left.\langle R\rangle=1-\rho \cdot K_{d}^{\prime} ; \theta\right\}$. and $\left(K_{d}\right\rangle=K_{d}^{G} \exp \left(\beta^{2} \sigma_{f}^{2}: 2\right)$. For noncorrelation between $\ln K_{d}$ and $\ln K_{v}$, we have

$$
\begin{gathered}
A_{: 2}=0 \\
A_{::=}=\int_{0}^{:}\left(1-\frac{1}{R !}\right)^{2}\left\{\exp \left[C_{d ! !}(\xi)\right]-1\right\} d \xi
\end{gathered}
$$

where $\left\langle K_{d}\right\rangle=K_{d}^{(i} \exp \left(\sigma_{d}^{2} 2\right)$.

In (40) the macrodispersivity $A_{z:}$ is expressed as the linear combination of three parts. $A_{z 1}, A_{z 2}$, and $A_{z 3} . A_{z 1}$ consists of three terms shown in (41). which represent the contributions to $A_{z=1}$ from the covariance of unsaturated hydraulic conductivity. the cross covariance of water content with unsaturated hydrau. lic conductivity. and the covariance of soil water content. $A_{z 2}$ relates to the cross covariance of retardation factor and unsaturated hydraulic conductivity. and $A_{z 3}$ relates to the covariance of retardation factor. For passive solute. $A_{z 2}$ and $A_{z 3}$ vanish. Because adsorption coefficient may be positively or negatively correlated with the soil saturated hydraulic conductivity. $A_{z 2}$ can be a positive or a negative contribution to $A_{z:}$. Therefore the correlation structure between $\ln K_{d}$ and $\ln K_{\text {s }}$ significantly affects the magnitude of macrodispersivity.

By substituting (31), (33), and (34) into (41), the following closed-form expression of $A_{z 1}$ is obtained (see appendix):

$$
\begin{aligned}
& A_{: 1}=\lambda_{f}\left\{\sum_{n=1}^{*} \sum_{k=11}^{n} \frac{C_{n}^{n} \sigma_{i}^{2 n-k^{\prime}} H^{2 k} \sigma_{u}^{2 k}}{[n-k(\rho-1)] n !}\right. \\
& \left.-\sum_{n=1}^{\infty} \sum_{k=11}^{n} \frac{C_{n}^{k} \sigma_{f}^{2 / n-k^{\prime}} H^{2 k} \sigma_{u}^{2 k}}{[n+k(\rho-1)] n !} \exp \left[-[n+k(\rho-1)] \frac{z_{r}}{\lambda_{f}}\right]\right\} \\
& +\frac{1}{\langle R\rangle^{2}}\left(\frac{\theta_{1}-\theta_{r}}{\langle\theta\rangle}\right)^{2} \exp \left(2 E+B^{2} \sigma_{u}^{2}\right) \frac{\lambda_{f}}{\rho}
\end{aligned}
$$

$$
\begin{aligned}
& \cdot\left[\sum_{n=1}^{\infty} \frac{\left(B^{2} \sigma_{a}^{2}\right)^{n}}{n n !}-\sum_{n=1}^{x} \frac{\left(B^{2} \sigma_{a}^{2}\right)^{n}}{n n !} \exp \left(-n \rho \frac{z_{c}}{\lambda_{f}}\right)\right] \\
& -\frac{2}{\langle R\rangle}\left(\frac{\theta_{s}-\theta_{r}}{\langle\theta\rangle}\right) \exp \left(E+\frac{1}{2} B^{2} \sigma_{a}^{2}\right) \frac{\lambda_{f}}{\rho} \\
& \cdot\left[\sum_{n=1}^{\infty} \frac{\left(-B H \sigma_{a}^{2}\right)^{n}}{n n !}-\sum_{n=1}^{\infty} \frac{\left(-B H \sigma_{a}^{2}\right)^{n}}{n n !} \exp \left(-n \rho \frac{z_{c}}{\lambda_{f}}\right)\right]
\end{aligned}
$$

where $C_{n}^{k}$ is the binomial coefficient and $\rho=\lambda_{f} / \lambda_{a}$.

Under the condition of perfect correlation between $\ln K_{d}$ and $\ln K_{s}, A_{z 2}$ and $A_{z 3}$ have the forms of

$$
\begin{aligned}
& A_{z: 2}=-2\left(1-\frac{1}{\langle R\rangle}\right)\left[\sum_{n=1}^{*} \frac{\left(\beta \sigma_{i}^{2}\right)^{n}}{n n !}-\sum_{n=1}^{*} \frac{\left(\beta \sigma_{t}^{2}\right)^{n}}{n n !} \exp \left(-n \frac{z_{c}}{\lambda_{t}}\right)\right] \lambda_{l} \\
& A_{z: 3}=\left(1-\frac{1}{\langle R\rangle}\right)^{2}\left[\sum_{n=1}^{*} \frac{\left(\beta^{2} \sigma_{!}^{2}\right)^{n}}{n n !}-\sum_{n=1}^{x} \frac{\left(\beta^{2} \sigma_{t}^{2}\right)^{n}}{n n !} \exp \left(-n \frac{z_{t}}{\lambda_{t}}\right)\right] \lambda_{t}
\end{aligned}
$$

For the noncorrelation hetween $\ln K_{d}$ and $\ln K_{s} . A_{z 2}$ and $A_{z 3}$ can be simplified to

$$
A: 2=0
$$

$$
A_{::}=\left(1-\frac{1}{\langle R\rangle}\right)^{2} \lambda_{d}\left[\sum_{n=1}^{\vdots} \frac{\left(\sigma_{d}^{2}\right)^{n}}{n n !}-\sum_{n=1}^{n} \frac{\left(\sigma_{i}^{2}\right)^{n}}{n n !} \exp \left[-\frac{\sum_{0}}{\lambda_{d}} n\right]\right]
$$

When $z_{c} / \lambda_{f} \ll 1$. the macrodispersivities are given by

$$
\begin{aligned}
A_{z:}= & \left\{\frac{1}{\langle R\rangle^{2}}\left(\frac{\theta_{s}-\theta_{r}}{\langle\theta\rangle}\right)^{2} \exp \left(2 E-B^{2} \sigma_{a}^{2}\right)\left[\exp \left(B^{2} \sigma_{a}^{2}\right)-1\right]\right. \\
& -\frac{2}{\langle R\rangle} \frac{\theta_{1}-\theta_{r}}{\langle\theta\rangle} \exp \left(E+\frac{1}{2} B^{2} \sigma_{a}^{2}\right)\left[\exp \left(-B H \sigma_{a}^{2}\right)-1\right] \\
& +\left[\exp \left(\sigma_{f}^{2}+H^{2} \sigma_{a}^{2}\right)-1\right]-2\left(1-\frac{1}{\langle R\rangle}\right) \\
& \left.\cdot\left[\exp \left(\beta \sigma_{f}^{2}\right)-1\right]+\left(1-\frac{1}{\langle R\rangle}\right)^{2}\left[\exp \left(\beta^{2} \sigma_{f}^{2}\right)-1\right]\right\} z_{c}
\end{aligned}
$$

$$
\begin{aligned}
A_{::=}= & \left\{\frac{1}{\langle R\rangle^{2}}\left(\frac{\theta_{s}-\theta_{r}}{\langle\theta\rangle}\right)^{2} \exp \left(2 E+B^{2} \sigma_{a}^{2}\right)\left[\exp \left(B^{2} \sigma_{a}^{2}\right)-1\right]\right. \\
& -\frac{2}{\langle R\rangle} \frac{\theta_{s}-\theta_{r}}{\langle\theta\rangle} \exp \left(E+\frac{1}{2} B^{2} \sigma_{a}^{2}\right)\left[\exp \left(-B H \sigma_{a}^{2}\right)-1\right] \\
& +\left[\exp \left(\sigma_{f}^{2}+H^{2} \sigma_{u}^{2}\right)-1\right]+\left(1-\frac{1}{\langle R\rangle}\right)^{2} \\
& \left.\cdot\left[\exp \left(\sigma_{d}^{2}\right)-1\right]\right\} z_{c}
\end{aligned}
$$

for the perfect correlation and noncorrelation between $\ln K_{d}$ and $\ln K_{s}$, respectively. Equations (51) and (52) indicate that 
the macrodispersivities are linear functions of the solute travel distance $z_{c}$. The difference of the macrodispersivities between negative and positive correlations can be evaluated by

$$
A_{: z}^{\mathrm{Neg}}-A_{: z}^{\mathrm{Pos}}=4\left(1-\frac{1}{\langle R\rangle}\right) \sinh \left(|\beta| \sigma_{f}^{2}\right) z_{\mathrm{c}}
$$

where $A_{z z}^{\mathrm{Neg}}$ and $A_{z z}^{\text {Pos }}$ are the macrodispersivities for negative and positive correlations between $\ln K_{d}$ and $\ln K_{s}$, respectively, and $\sinh (x)$ is the hyperbolic sine function.

For the case of $z_{c} / \lambda_{f} \gg 1$ and perfect correlation between $\ln K_{s}$ and $\ln K_{d}$, the macrodispersivity tends to a constant

$$
\begin{aligned}
A_{: z}= & \left\{\frac { 1 } { \rho \langle R \rangle ^ { 2 } } ( \frac { \theta _ { v } - \theta _ { r } } { \langle \theta \rangle } ) ^ { 2 } \operatorname { e x p } ( 2 E + B ^ { 2 } \sigma _ { u } ^ { 2 } ) \left[E i\left(B^{2} \sigma_{u}^{2}\right)-C\right.\right. \\
& \left.-\ln \left(B^{2} \sigma_{u}^{2}\right)\right]-\frac{2}{\rho\langle R\rangle} \frac{\theta_{s}-\theta_{r}}{\langle\theta\rangle} \exp \left(E+\frac{1}{2} B^{2} \sigma_{u}^{2}\right) \\
& \cdot\left[E i\left(-B H \sigma_{u}^{2}\right)-C-\ln \left(|B H| \sigma_{u}^{2}\right)\right] \\
& +\sum_{n=1}^{x} \sum_{k=11}^{n} \frac{C_{n}^{k} \sigma_{f}^{2}(n-k) H^{2 k} \sigma_{u}^{2 k}}{[n+k(\rho-1)] n !}-2\left(1-\frac{1}{\langle R\rangle}\right)^{2} \\
& \cdot\left[E i\left(\beta \sigma^{2}\right)-C-\ln \left(|\beta| \sigma_{i}^{2}\right)\right]+\left(1-\frac{1}{\langle R\rangle}\right)^{2} \\
& \left.\cdot\left[E i\left(\beta^{2} \sigma^{2}\right)-C-\ln \left(|\beta| \sigma_{i}^{2}\right)\right]\right\}
\end{aligned}
$$

Here $E i(x)$ is the exponential integration. and $C=0.577 \ldots$ is Euler's constant. The difference of the macrodispersivity between negative and positive correlations is

$A_{::}^{\nu_{\mathrm{eg}}}-A_{::}^{\mathrm{P}_{i x}}=2\left(1-\frac{1}{(R)}\right)\left[E_{i}\left(\beta \sigma_{f}^{2}\right)-E i\left(-\beta \sigma_{t}^{2}\right)\right] \lambda$.

\section{Discussion}

The main purpose of this study was to relate the longitudinal macrodispersivity to the statistical parameters of the unsaturated soil and chemical heterogeneity properties under the condition of gravity-dominated unsaturated flow. The general results of this study are illustrated in (46) to (50). Some results based on the analytical solutions are discussed as follows.

\subsection{Asymptotic Behavior of Macrodispersivity}

For short solute travel time, the macrodispersivity was proportional to the solute travel distance as indicated in (51) and (52). This short time characteristic of the macrodispersivity of adsorbing solute transport in the unsaturated soil was similar to that in the saturated soil studied by Bellin et al. [1993]. For the comparison of the present solution with Bellin et al.'s solution for the saturated situation, letting $H=0$ and $\beta= \pm 1$ in (51), we obtained

$$
\begin{aligned}
A_{::=}= & \left\{e^{\sigma_{f}^{2}}-1-2\left(1-\frac{1}{\langle R\rangle}\right)\left(e^{ \pm \sigma_{f}^{2}}-1\right)\right. \\
& \left.+\left(1-\frac{1}{\langle R\rangle}\right)^{2}\left(e^{\sigma_{f}^{2}}-1\right)\right\} z_{c}
\end{aligned}
$$

Bekelin et al.'s [1993] solution for short time is

$$
\begin{aligned}
A_{z:=} & \left\{\frac{8}{15} \sigma_{i}^{2} \pm \frac{-4}{3}\left(1-\frac{1}{\langle R\rangle}\right) \sigma_{i}^{2}\right. \\
& \left.+\left(1-\frac{1}{\langle R\rangle}\right)^{2}\left(e^{\sigma_{i}^{2}}-1\right)\right\} z_{r}
\end{aligned}
$$

The macrodispersivity evaluated from (56) was slightly larger than that from (57) for the short travel time. This difference was stemmed from the assumption of neglecting the perturbation of the hydraulic gradient during the derivation of (51).

When $z i \dot{\lambda} \gg 1$, the macrodispersivity was expressed by (54), a linear function of the correlation length of $\ln K_{s}$. Here. $A_{z:}$ depended on the statistical parameters of the soil. the adsorption parameters of the solute, and the soil saturation. As a special case of the saturated situation, i.e., $H=0$, the first and second terms on the right-hand side of (54) vanished. and the general solution was reduced to

$$
\begin{aligned}
A_{::=} & \left\{\left(\sigma_{i}^{2}+\frac{\sigma_{f}^{4}}{4}+\frac{\sigma_{f}^{\circ}}{18}+\cdots\right)-2\left(1-\frac{1}{\langle R\rangle}\right)\right. \\
& \cdot\left(\beta \sigma_{f}^{2}+\frac{\beta^{2} \sigma_{f}^{+}}{4}+\frac{\beta^{3} \sigma_{f}^{\circ}}{18}+\cdots\right) \\
& \left.+\left(1-\frac{1}{\langle R\rangle}\right)^{2}\left(E i\left(\sigma_{i}^{-}\right)-C-\ln \sigma_{i}^{2}\right)\right\} \lambda_{f}
\end{aligned}
$$

By neglecting the high-order terms of $\sigma_{f}^{2}$ and taking $\beta= \pm 1$, (58) is the same as Bellin et al.'s [1993] solution

$$
\begin{aligned}
A_{::=} & \left\{\sigma_{i}^{2}=2\left(1-\frac{1}{\langle R\rangle}\right) \sigma_{i}^{2}\right. \\
& \left.+\left(1-\frac{1}{\langle R\rangle}\right)^{2}\left(E i\left(\sigma_{f}^{2}\right)-C-\ln \sigma_{f}^{2}\right)\right\} \lambda_{l}
\end{aligned}
$$

For nonreactive solute. $R=1,(58)$ is reduced to

$$
A_{: z}=\left(\sigma_{i}^{2}+\frac{\sigma_{f}^{+}}{4}+\frac{\sigma_{f}^{\dagger}}{18}+\cdots\right) \lambda_{f}
$$

which has the same form as Dagan's [1984] solution when neglecting the high order terms of $\sigma_{f}^{2}$. In Figure 1, the curren: solutions are compared with Bellin et al.'s [1993] solution for adsorbing solute and the Dagan's [1984] solution for nonadsorbing solute in saturated soils. The agreement of our resuits with those of Dagan and Bellin et al. was very good when our solutions were simplified to the saturated situation.

\subsection{Effects of $\boldsymbol{\sigma}_{f}^{2}, \boldsymbol{\sigma}_{a}^{2},\langle\boldsymbol{\theta}\rangle$, and Water Content Variability on Macrodispersivity}

The coefficient of variation of the unsaturated hydraulic conductivity, $\mathrm{CV}_{K}$, could be determined from (24) and (32) as

$$
\mathrm{CV}_{K}=\left[\exp \left(\sigma_{f}^{2}+H^{2} \sigma_{u}^{2}\right)-1\right]^{1 / 2}
$$

The variability of unsaturated soil was related to $\left(\sigma_{f}^{2}+H^{2} \sigma_{a}^{2}\right)$, which displayed combined effects of the soil variabilities and hydraulic properties. The effect of variability of $\alpha$ on the unsaturated soil variability was related to soil water content. The macrodispersivity $A_{z z}$ versus the scaled solute travel distance. $z_{c} / \lambda_{f}$, is plotted in Figure 2 for different values of $\sigma_{f}^{2}$ and $\mathrm{CV}_{a}=\sigma_{a} / A$. It is shown that $A_{z z}$ increases with the increasing $\sigma_{f}^{2}$ and $\mathrm{CV}_{a}$ because the variability of the unsaturated hydraulic conductivity increases with the increases of $\sigma_{f}^{2}$ and $\mathrm{CV}_{a}$.

Figure 3 shows the effect of water content variability on the 


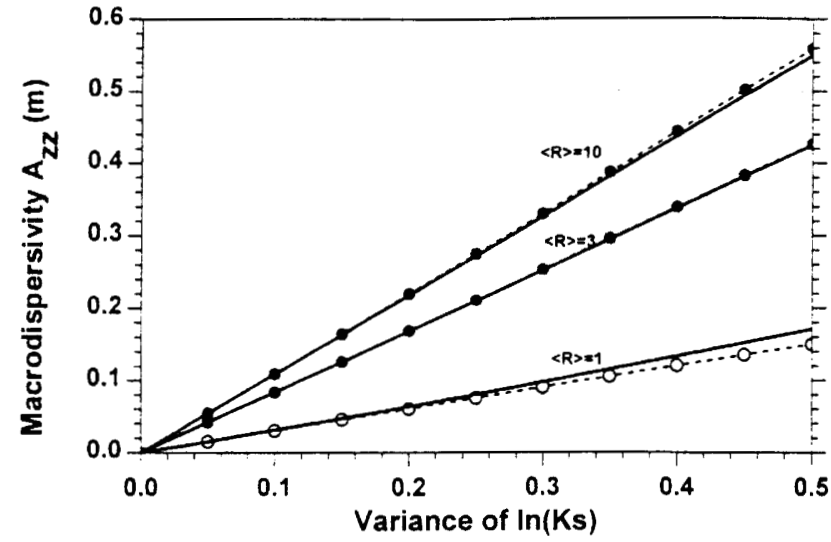

Figure 1. Macrodispersivities calculated by the current solution (solid lines) for different retardation factors and by the solutions of Dagan [1984] (open circles) and Bellin et al. [1993] (solid circles) for saturated water flow.

longitudinal macrodispersivity for different mean values of water content. The effect of water content variability on $A_{z z}$ increased with decreasing mean water content. This was physically meaningful because the variabilities of water content and soil water velocity increased with the decrease of water content. The effect of water content variability on longitudinal macrodispersivity was due to the cross covariance of water content with unsaturated hvdraulic conductivity and the covariance of water content in $(\$ 1)$. The calculated results showed that the cross covariance of water content with the unsaturated hydraulic conductivity was much larger than the covariance of water content. Furthermore. the correlation of water content with the unsaturated hydraulic conductivity was positive. and consequently $C_{H K^{\prime}}(\mathbf{r})$ was larger than zero. Therefore macrodispersivity was smaller for the case of considering the water content variability than for the case of neglecting it because $C_{H \mathcal{K}}(\mathbf{r})$ was a negative contribution to the macrodispersivity.

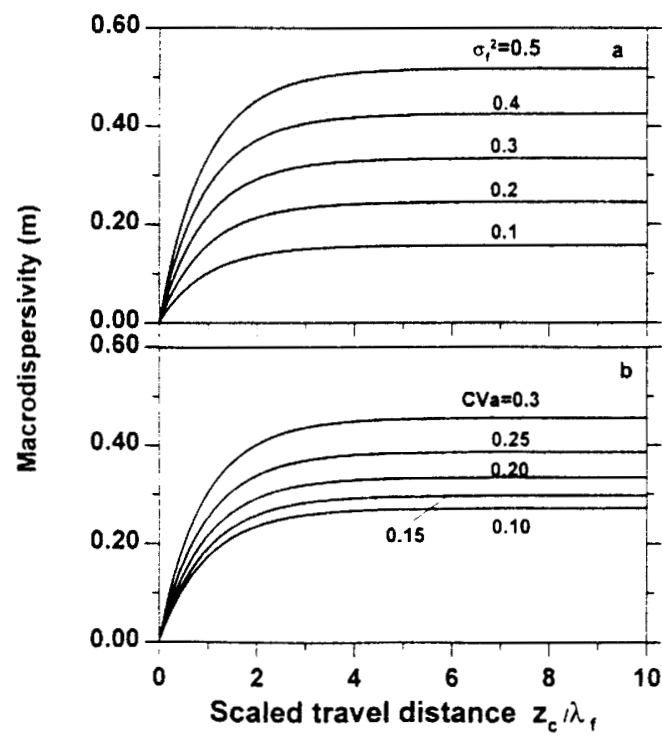

Figure 2. Macrodispersivities versus the scaled solute travel distance for (a) different values of $\sigma_{f}^{2}$, and (b) different vałues of $\mathrm{CV}_{a}$, for $\langle R\rangle=3.0,\langle\theta\rangle=0.3, \lambda_{f}=\lambda_{a}=0.3 \mathrm{~m}$. and $\beta=-1$.

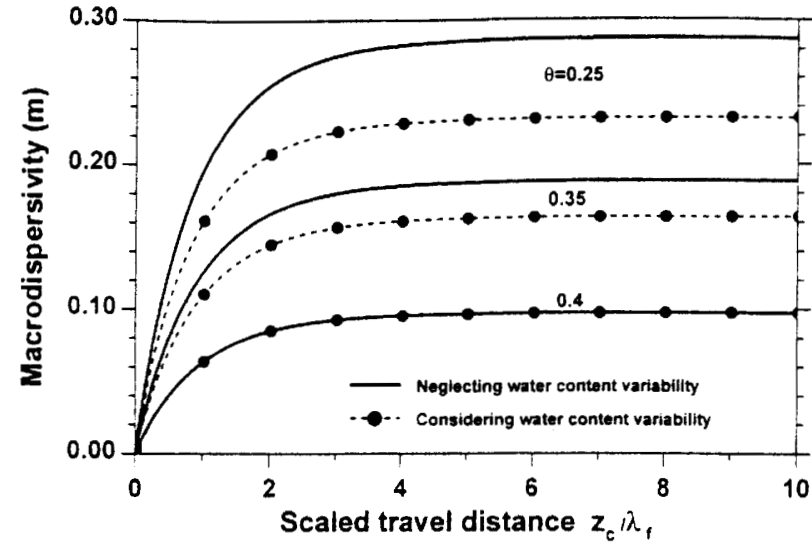

Figure 3. Macrodispersivities versus the scaled solute travel distance with and without considering the water content variability. for $\langle R\rangle=1 . \sigma_{f}^{2}=0.3, \mathrm{CV}_{a}=0.2 . \lambda_{f}=\lambda_{a}=0.3$ $\mathrm{m}$. and $\beta=-1$.

For soils with low water content. neglecting the water content variability would result in a large error in the evaluation of macrodispersivity.

The dependence of $A_{z:}$ on the water content is shown in Figure + . The macrodispersivity increased with decreasing water content. For comparison. the analytical expression of $A=$ for steady. saturated water flow and adsorbing solute derived by Bellin et al. [1993] is also depicted in Figure 4. When water content tends to saturation. macrodispersivity calculated by (46) $-(48)$ has a reasonable agreement with that of Bellin et al. [1993].

\subsection{Effect of Retardation Factor and Correlation Structure on Macrodispersivity}

For small and large solute travel distances. the differences of macrodispersivity between negative and positive correlations between $\ln K_{d}$ and $\ln K$, were given by (53) and (55), respectively. The differences did not depend on the soil saturation. which was the direct results of assuming $K_{d}$ to depend on $K$, only and not to depend on the water content. Because sinh $(x)$ in (53) was an increasing function and $E i(x)>E i(-x)$ in (55)

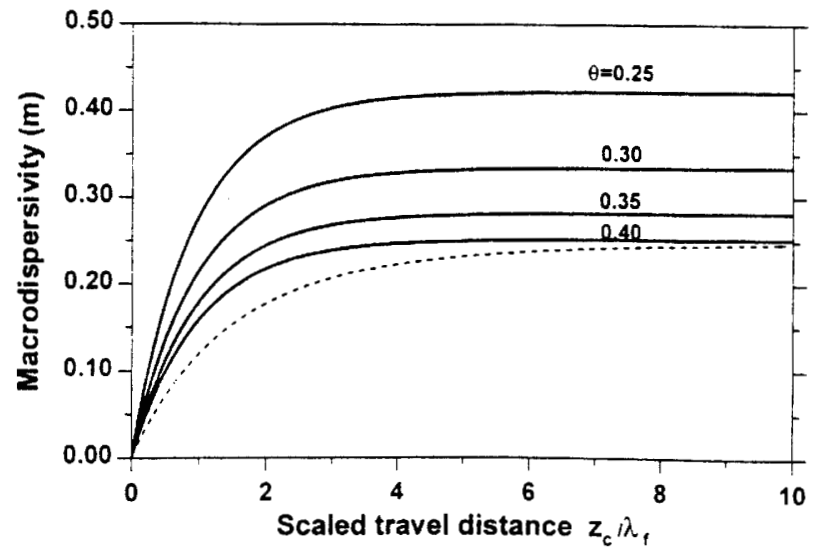

Figure 4. Macrodispersivities versus the scaled solute travel distance for different water contents, for $\langle R\rangle=3, \sigma_{f}^{2}=0.3$. $C V_{d}=0.2 . \lambda_{f}=\lambda_{a}=0.3 \mathrm{~m}$, and $\beta=-1$. The dashed line represents the solution of Bellin et al. [1993] for saturated water flow. 


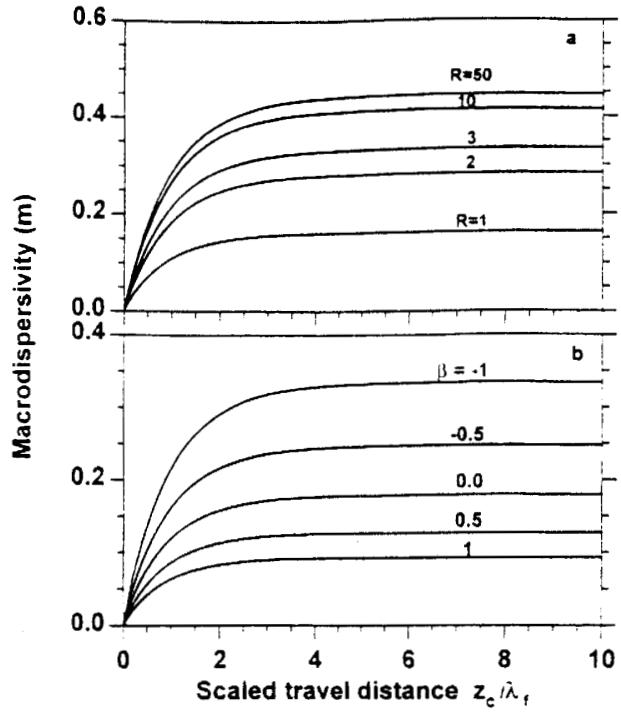

Figure 5. Macrodispersivities versus the scaled solute travel distance for (a) different retardation factors and (b) different correlation structures, for $\langle\theta\rangle=0.3, \sigma_{f}^{2}=0.3, \mathrm{CV}_{a}=0.2$, and $\lambda_{f}=\lambda_{d}=0.3 \mathrm{~m}$.

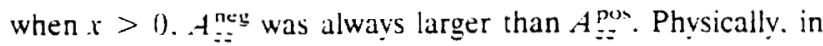
the case of negative correlation between $\ln K_{d l}$ and $\ln K_{s}$. solute spreading was enhanced compared with the positively correlated situation.

The macrodispersivity versus the scaled solute travel distance for different values of $\langle R\rangle$ and $\beta$ is shown in Figures 5 a and $5 \mathrm{~b}$. respectively. The macrodispersivity was enhanced by the chemical heterogeneity. Even with the variability of adsorption coefficient kept as a constant. macrodispersivity increased with increasing mean retardation factor. This result is shown clearly by $(+8)$ and $(50)$, in which $A_{z}$ is proportional to $(1-1 /\langle R\rangle)^{2}$. It is worth notice that $A_{z:}$ was much sensitive to small retardation factors.

The macrodispersivity was smaller for the positive correlated case than for the negative case (Figure $5 \mathrm{~b}$ ). The absolute value of $\beta$, which expressed the change rate of $\ln K_{d}$ with the change of $f$. played a very important role in the determination of macrodispersivity. It is obvious in (46)-(48) that $A_{z=1}$ and $A_{z=3}$ are not affected by the correlation structures between $\ln K_{a}$ and $\ln K_{r}$. The foremost impact of the correlation structure on the behavior of macrodispersivity was caused by the interaction between the perturbations of water flux and retardation factor, which was expressed by $A_{z 2} . A_{z}$ was less than zero for positive correlation, and greater than zero for negative correlation. The larger the absolute value of $\beta$ was, the larger the absolute value of $A_{z 2}$.

\section{Conclusions}

An analytical solution of macrodispersivity was derived for adsorbing solute transport in heterogeneous unsaturated soils under assumptions of gravity-dominated flow and negligible local dispersion. The unsaturated hydraulic conductivity and the water content were treated as spatial random functions. The chemical heterogeneity was represented by an adsorption coefficient which was assumed as a spatial random function with specified correlation structures. The perfect correlation and noncorrelation of the adsorption coefficient with the saturated hydraulic conductivity were considered.

The closed-form expression of the macrodispersivity was expressed as a function of the hydraulic and adsorbing statistical properties of the unsaturated soil. The general solution can be simplitied to special cases, such as to the saturated soil and the nonadsorbing solute. The current solutions had an excellent agreement with the available solutions of Dagan [1984] and Bellin et al. [1993] for the special cases. For the short solute travel distance, the macrodispersivity linearly increased with the increase of the solute travel distance. The macrodispersivity tended to a constant when the center of the solute plume moved a couple of tenths of a correlation length of the saturated hydraulic conductivity. The macrodispersivity increased with decreasing water content and increasing variances of $\ln K_{s}$ and $\alpha$. Neglecting the variability of water content would overestimate the macrodispersivity, especially for low water content. The correlation structure between adsorption coefficient and saturated hydraulic conductivity had significant impacts on macrodispersivity. Negative correlation between in $K_{d}$ and $\ln K_{s}$ enhanced the solute spreading compared with the positive correlation and noncorrelation. The macrodispersivity increased with increasing retardation factor.

\section{Appendix}

A general form of the integration in $(41)$ is

$$
\begin{aligned}
I=\int_{11}^{:}\left\{\operatorname { e x p } \left[\sigma_{i}^{2} \exp \left(-\frac{r}{\lambda_{l}}\right)\right.\right. \\
\left.\left.+H^{2} \sigma_{a}^{2} \exp \left(-\frac{r}{\lambda_{u}}\right)\right]-1\right\} d r
\end{aligned}
$$

By setting $\xi=\exp \left(-r / \lambda_{f}\right), \rho=\lambda_{f} / \lambda_{u}$, and expanding the resulting integrand into an exponential series, (A1) becomes

$$
I=\lambda_{f} \int_{e=1}^{i} \frac{1}{\xi} \sum_{n=1}^{\infty} \frac{\left(\sigma_{f}^{2} \xi+H^{2} \sigma_{a}^{2} \xi^{\rho}\right)^{n}}{n !} d \xi
$$

Using the binomial expansion in (A2), we have

$$
I=\lambda_{f} \int_{e: 1}^{1}\left\{\sum_{n=1}^{x} \sum_{k=1)}^{n} \frac{1}{n !} C_{n}^{k} \sigma_{f}^{2(n-k)}\left(H \sigma_{a}\right)^{2 k} \xi^{n+k(p-1)-1}\right\} d \xi
$$

where $C_{n}^{k}$ is the binomial coefficient. Because $n+k(\rho-1)$ $-1 \neq-1$, integrating term by term in (A3) leads to

$$
\begin{aligned}
I= & \lambda_{f} \sum_{n=1}^{\infty} \sum_{k=0}^{n} \frac{C_{n}^{k} \sigma_{f}^{2(n-k)}\left(H \sigma_{u}\right)^{2 k}}{[n+k(\rho-1)] n !} \\
& \cdot\left\{1-\exp \left(-[n+k(\rho-1)] \frac{z_{c}}{\lambda_{f}}\right)\right\}
\end{aligned}
$$

Acknowledgment. This research was supported through the NSFEPSCOR ADP grant of the University of Wyoming, OSR9108774.

\section{References}

Aris, R., On the dispersion of a solute in a fluid flowing through a tube, Proc. R. Soc. London A, 235, 67-78, 1956. 
Bellin, A.. A. Rinaldo, W. J. P. Bosma, S. E. A. T. M. van der Zee. and $Y$. Rubin. Linear equilibrium adsorbing solute transport in physically and chemically heterogeneous porous formations, 1 , Analytical solutions. Water Resour. Res., 29, 4019-4030, 1993.

Bosma, W. J. P.. A. Bellin, S. E. A. T. M. van der Zee, and A. Rinaldo, Linear equilibrium adsorbing solute transport in physically and chemically heterogeneous porous formations, 2, Numerical results, Water Resour. Res.. 29, 4031-4043, 1993.

Christakos, G.. Random Field Models in Earth Sciences, Academic, San Diego, Calif.. 1992.

Dagan, G., Stochastic modeling of groundwater flow by unconditional and conditional probabilities, 2, The solute transport, Water Resour. Res., 18, S35-848, 1982.

Dagan, G.. Solute transport in heterogeneous porous formations, $J$. Fluid Mech.. 145, 151-177, 1984.

Destouni, G.. and V. Cvetkovic, Field scale mass arrival of sorptive solute into the groundwater. Water Resour. Res., 27. 1315-1325, 1991.

Freeze, R. A.. A stochastic-conceptual analysis of one-dimensional groundwater flow in nonuniform homogeneous media, Water Resour. Res., 11. 725-741. 1975.

Frevberg. D. L.. A natural gradient experiment on solute transport in a sand and gravel aquifer, 2. Spatial moments and the advection and dispersion of nonreactive tracers, Water Resour. Res., 22, 2031-2046, 1986.

Gardner, W. R., Some steady state solutions of unsaturated moisture flow equations with application to evaporation from a water table. Soil Sci.. 85. 228-232, 1958.

Gelhar, L. W.. and C. L. Axness. Three-dimensional stochastic analysis of macrodispersion in aquifers. Water Resour. Res.. 19, 161-180. 1983.

Kabala. Z. J., and G. Sposito, A stochastic mudel of reactive solute transport with time-varying velocity in a heterogeneous aquifer, $\mathrm{Wa}$ ter Resour. Res., 27, 341-350, 1991.

Roberts. P. V., M. N. Goltz, and D. M. Mackay, A natural gradient experiment on solute transport in a sand aquifer, 3, Retardation estimates and mass balances for organic solutes, Water Resour. Res., 22, 2047-2058. 1986.

Russo, D.. Determinating soil hydraulic properties by parameter estimation: On the selection of a model for the hydraulic properties, Water Resour. Res., 24, 453-459, 1988.

Russo, D.. Stochastic analysis of simulated vadose zone solute transport in a vertical cross section of heterogeneous soil during nonsteady water flow, Water Resour. Res.. 27. 267-283. 1991.

Russo, D.. Stochastic modeling of macrodispersion for solute transport in a heterogeneous unsaturated porous formation. Water Resour. Res., 29, 383-397, 1993.

Russo, D.. and M. Bouton, Statistical analysis of spatial variability in unsaturated parameters, Water Resour. Res., 28. 1911-1925, 1992.

Sudicky. E. A., A natural gradient experiment on solute transport in a sand and gravel aquifer, Spatial variability of hydraulic conductivity and its role in the dispersion process, Water Resour. Res., 22, 20692082. 1986

Yeh. T.-C. J., L. W. Gelhar, and A. L. Gutjahr, Stochastic analysis of unsaturated flow in heterogeneous soils. 1. Statistically isotropic media. Water Resour. Res., 21, 447-456, 1985.

J. Wu, J. Yang, and R. Zhang. Department of Plant, Soil, and Insect Sciences. College of Agriculture, University of Wyoming, Laramie, WY 82071.

(Received April 28. 1995: revised Octuber 27, 1995: accepted November 13. 1995.) 